\title{
Post-streptococcal Reactive Arthritis with Neuropsychiatric Syndrome in adult (A case report with brief review of literature)
}

\author{
Dr. Mahendra Wawhal ${ }^{1}$, Dr. K.M.Raul ${ }^{2}$, Dr. S.H.Talib ${ }^{3}$, Dr. Sharad Garudkar ${ }^{4}$, \\ Dr. Ronak Modi ${ }^{5}$, Dr. Wajed Mogal ${ }^{5}$ \\ ${ }^{1}$ Associate Professor, ${ }^{2}$ Professor, ${ }^{3}$ Professor \& Head, ${ }^{4}$ Chief Resident, ${ }^{5}$ Senior Resident In Medicine, \\ Department of Medicine, Mahatma Gandhi Mission's Medical College \& Hospital, CIDCO, N-6, Aurangabad, \\ Maharashtra., India - 431003
}

\begin{abstract}
We report a case of Reactive Arthritis with Neuropsychiatric Syndrome in an adult associated with Group A beta haemolytic streptococcal infection ". "PANS" (Paediatric Acute Onset Neuropsychiatric Syndrome) is an immune mediated neuropsychiatric syndrome secondary to Group A beta haemolytic streptococcal infection which includes usually abrupt onset of obsessive-compulsive disorder, often pre-pubertal in onset, separation anxiety \& abnormal movements during attack ${ }^{2}$. PANS is very common in children especially pre-pubertal age group. However the existence of neuropsychiatric syndrome as a post-streptococcal manifestation in adults is rare \& long debated. There has been only one case report in the literature of poststreptococcal neuropsychiatric syndromes in adults ${ }^{3,19}$. We discuss the aspects of immune mediated poststreptococcal neuropsychiatric syndrome in adult.
\end{abstract}

Keywords: OCD (Obsessive Compulsive Disorder), PANS (Paediatric Acute Onset Neuropsychiatric Syndrome), PANDA (Paediatric Autoimmune Neuropsychiatric Disorders Associated with Streptococcal infections), PITANDS (Paediatric Infection-Triggered Autoimmune Neuropsychiatric Disorders), SC (Sydenham's chorea)

\section{Introduction}

PANS (Paediatric Acute Onset Neuropsychiatric Syndrome) usually occurs in children before puberty secondary to the Group A beta haemolytic streptococcal infection ${ }^{2}$. PANS was formerly known as "PANDAS" (Paediatric Autoimmune Neuropsychiatric Disorders Associated with Streptococcal infections) \& "PITANDS" (Paediatric Infection-Triggered Autoimmune Neuropsychiatric Disorders). The term PITANDS was used to reflect the variety of infectious organisms like streptococci $(81 \%) \&$ other infections (19\%) like influenza, Mycoplasma, Varicella (chickenpox) \& Lyme disease ${ }^{1,4,5}$, that had been associated with immune mediated neuropsychiatric syndrome ${ }^{1,2,4}$. PANS occurs mostly before puberty because by the age of puberty most of the population will have "immunity" to Group A beta haemolytic streptococcal infections.

There has been only one reported case of PANS beyond puberty i.e. in adults across world medical literature ${ }^{3}$. Cases of PANS can occur after puberty, because rheumatic fever and Sydenham's chorea have been documented in military personnel's and students living in crowded areas. Thus, PANS usually has onset before puberty but can occur at or after puberty, although manifesting with variable neuropsychiatric symptoms.

\section{Case History}

A 21 years old female patient had history of on and off multiple large \& small joint pains since four years when she was of 17 years of age. She had history of mood swings, over-sleepiness, depression, anxiety, emotional lability, dis-interest in the surroundings consistently since last four years with exacerbations intermittently. She was on regular treatment under a psychiatrist $\&$ was being treated as a case of depression. Patient was taking NSAIDS (Non-Steroidal Anti-Inflammatory Drugs) for joint pains as \& when required.

On presentation to this hospital patient had severe joint pains involving large \& small joints associated with above said psychiatric complaints, for which she was admitted in psychiatry ward. She had no history of involuntary movements. Treatment started with Selective Serotonin Reuptake Inhibitors (SSRI) \& symptomatic treatment with analgesics for joint pain. Patient had no relief in joint pains; hence the patient was accepted transfer in medicine ward.

On clinical examination patient was conscious, oriented but irritable. She was afebrile, her pulse rate was 106/min regular, BP-130/76 mm of $\mathrm{Hg} \&$ rest of the general examination was normal. There was no signs of rheumatic fever. Systemic examination including CNS examination was normal. However she had the above said psychiatric complaints.

Patients investigations were as follows- Hb-12.4 gm\%, WBC count-6800 $/ \mathrm{mm}^{3}$ (N-72\%, L-17\%, M$4 \%$, E-5\%, B-0\%), Platelet count-215000/ $\mathrm{mm}^{3}$, Blood Urea-21, Serum creatinine- 0.8 , Serum sodium-138, 
Serum potassium-4.2, Total bilirubin-0.8, Direct bilirubin-0.3, SGOT-26, SGPT-30, Alkaline phosphatise-58, Serum calcium-9.4, Serum uric acid-4.4 mg/dl, ESR-54 mm, Rheumatoid factor titre(RA Factor)-26 U/ML(Normal), C-Reactive Protein(CRP)-284 mg/L(High), Anti-Steptolysin O (ASO) Titre > 400 (High), ANA \& ds-DNA was negative. Patients throat swab culture was negative for Group A beta haemolytic streptococcus, however three blood cultures were negative for three samples. ECG was normal. 2D Echo was normal with no evidence of cardiac involvement. X-Ray of large joints showed non-erosive arthritis with juxtaarticular osteopenia.

From the investigations it was clear that the aetiology of joint pain was post-streptococcal immune mediated reactive arthritis. Obsessive compulsive disorder was shown to be associated with Post-streptococcal autoimmune syndrome in pre-pubertal children's, called as PANS (Paediatric Acute Onset Neuropsychiatric Syndrome). But this patient was of 21 years of age \& she had onset of neuropsychiatric symptoms at the age of 17 years. There are very few case reports in literature about PANS presenting beyond puberty.

Patient was treated as a case of post-streptococcal reactive arthritis with neuropsychiatric syndrome. She was put on salicylates (Aspirin) in adequate doses- $100 \mathrm{mg} / \mathrm{kg}$ body weight/day for one month and then tapered and stopped in three months. She was given stat dose of Benzathine penicillin (Penidura) prophylaxis 1.2 MU intramuscularly \& three weekly injection penidura was advised. After giving salicylates the patient responded briskly \& was symptom free in one week. Her psychiatric complaints also diminished to the extent that all her prior psychiatric medicines were methodically omitted except $0.5 \mathrm{mg}$ of clonazepam h.s. for sometime. In course of illness no steroids were given to the patient. The patient is in regular follow up with no arthralgias, arthritis or any neuropsychiatric symptoms. Due to prompt \& robust response to Aspirin the diagnosis of post-streptococcal reactive arthritis with neuropsychiatric syndrome stood confirmed. Secondly we want to emphasize the fact that the post-streptococcal neuropsychiatric syndrome responded briskly to salicylates. In other words, in a given case, with a history, clinical features, laboratory findings suggestive of post-streptococcal neuropsychiatric syndrome, a good response to aspirin, may be taken as the confirmation of diagnosis as other conditions like SLE which is the closest differential will not respond to aspirin.

\section{Discussion}

In paediatric age group, the term PANS (Paediatric Acute Onset Neuropsychiatric Syndrome) is defined as abrupt onset of obsessive-compulsive disorder (OCD) with various neuropsychiatric symptoms occurring in episodic course secondary to immunological response to Group A beta haemolytic streptococcal infection. PANS may be associated with various neuropsychiatric symptoms i.e. emotional liability, change in school performance, personality change, bedtime fears, separation anxiety, irritability, tactile or sensory defensiveness, impulsivity or distractibility, deterioration in handwriting, deterioration in mathematics skills, nightmares 2. PANS was formerly known as "PANDAS" (Paediatric Autoimmune Neuropsychiatric Disorders Associated with Streptococcal infections) ${ }^{1}$. The term "PITANDS" (Paediatric Infection-Triggered Autoimmune Neuropsychiatric Disorders) was used for various infectious organisms associated with immune mediated neuropsychiatric syndrome ${ }^{1}$. PITANDS can occur following infections with streptococci $(81 \%) \&$ other infections (19\%) like influenza, Mycoplasma, Varicella (chickenpox) \& Lyme disease ${ }^{\mathbf{1}, \mathbf{4}}$.

It is very difficult to isolate streptococcal organism as OCD \& Sydenham's chorea may occur after some months or longer following streptococcal infection. Whereas positive cultures and elevated antibody titres may be completely unrelated to the neuropsychiatric symptoms, since Group A streptococcal infections are such a common occurrence among children. Also other problems are need for an appropriately performed throat culture; the lack of sensitivity of rapid strep tests and throat cultures (5-15\% false negatives), the presence of a carrier state among 1 in 20 children, and the need for serial measurements of anti-streptolysin O (ASO) titre at specified intervals in order to document an immune response to an acute infection ${ }^{6}$. The organism (Group A beta haemolytic streptococcus) may no longer be present when symptoms appear. Hence isolation of streptococci is excluded from the PANS criteria.

Clinical Features: Clinical criteria for PANS ${ }^{1}$ :

A. Abrupt, dramatic onset of obsessive-compulsive disorder or severely restricted food intake

B. Concurrent presence of additional neuropsychiatric symptoms, with similarly severe and acute onset, from at least two of the following seven criteria:
a. Anxiety
b. Emotional lability and/or depression
c. Irritability, aggression and/or severely oppositional behaviours
d. Behavioural (developmental) regression
e. Deterioration in school performance
f. Sensory or motor abnormalities
g. Somatic signs and symptoms, including sleep disturbances, enuresis or urinary frequency 
C. Symptoms are not better explained by a known neurologic or medical disorder, such as Sydenham' chorea.

The term "paediatric onset" in PANS is defined in many different ways as before age 18 years or age 21 years. Rheumatic fever is most common in 5 to 15 year-old children, though it can develop in younger children and adults. PANS is not associated with other manifestations of acute rheumatic fever, while Sydenham's chorea is associated with other manifestations of acute rheumatic fever. Clinical Sydenham's chorea and PANS are similar in many aspects; both have antibodies against basal ganglia of brain ${ }^{14}$. But PANS is not equal to a "mild case of Sydenham's Chorea", as the presence of chorea, rheumatic carditis or any of the other major manifestations of rheumatic fever is an exclusion criterion for PANS ${ }^{1,2}$. Obsessive-compulsive symptoms occur in seventy five percent of Sydenham's chorea patients \& begin 2-4 weeks before the onset of the Sydenham's chorea, so OCD may occur secondary to streptococcal infections without Sydenham's chorea 12 .

\section{Pathophysiology:}

The aetiology of PANS is controversial. A hypothesis for OCD \& tics in PANS is similar to that of Sydenham's chorea of rheumatic fever, an autoimmune disorder triggered by streptococcal infection, where antibodies attack the brain and cause neuropsychiatric symptoms ${ }^{7,8}$.

The molecular mimicry hypothesis is a proposed mechanism for PANS ${ }^{9}$. This hypothesis shows that antigens (M protein) on the cell wall of the streptococcal bacteria are similar in molecular level to the proteins of the heart valve, joints \& brain. M protein is a macromolecule which is incorporated in fimbriae present on the cell membrane projecting on the bacterial cell wall. It is the major virulence factor \& also basically responsible for antigenic shift and antigenic drift among Group A streptococci ${ }^{10,11}$. In a typical bacterial infection, body produces antibodies against the infecting bacteria, and the antibodies helps to eliminate the bacteria from the body. In some rheumatic fever patients, auto-antibodies may attack patient's own heart tissue, leading to carditis, or cross-react with joints, leading to arthritis and/or cross react with basal ganglia causing abnormal movements known as Sydenham's chorea or "St. Vitus' Dance" 12. In PANS the basal ganglia of brain particularly caudate nucleus \& putamen is affected, which is believed to be responsible for movement and behaviour ${ }^{12,13}$.

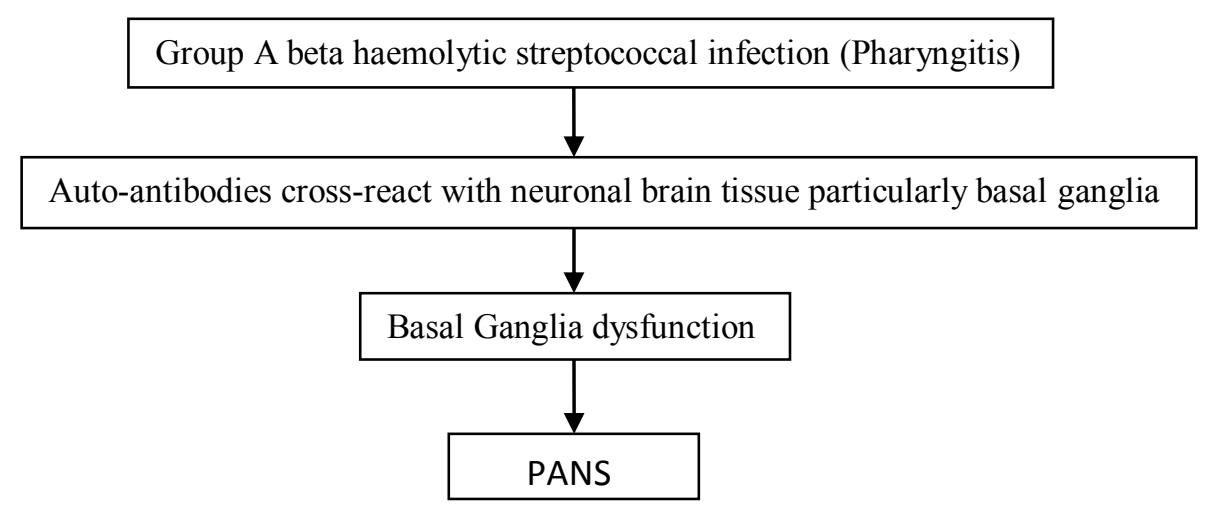

Fig. 1 Pathophysiology of Post-streptococcal autoimmune neuropsychiatric disorder ${ }^{12}$

\section{Treatment:}

The prevention of streptococcal infections with penicillin or Azithromycin was associated with decreased numbers of neuropsychiatric symptom exacerbations and overall improvements in symptom severity ${ }^{15}$. Antibiotics prophylaxis is required for all cases of rheumatic fever, including those presenting only with chorea. In contrast, long term antibiotics prophylaxis is generally not recommended for PANS although some small sample size studies shown beneficial effects \& there is need for long term studies ${ }^{16,17}$. Intravenous immunoglobulin (IVIG) \& Plasmapheresis is the more immediate and effective option in acute exacerbation of PANS but is most expensive and invasive treatment option ${ }^{18}$. Most clinicians do not initially start with SSRIs in treatment of PANS. However, as the disease progresses, they may find relief with the use of SSRIs. Cognitive behavioural therapy is found very helpful in management of PANS. Role of steroids \& NSAIDs in PANS is controversial \& associated with more side effects. 


\section{Conclusion:}

The existence of post-streptococcal neuropsychiatric syndrome is an accepted fact in the paediatric age group. However, in view of rampant streptococcal infection in developing countries like India where poverty \& poor sanitation are common, the association of Group A beta haemolytic streptococcal infection in adults is not uncommon. Although almost $98 \%$ of the population may be immune by the age of puberty, there can be a minority of adult cases which can have post-streptococcal immune-mediated neuropsychiatric features which are different from paediatric age group such as there may be no tics, obsessions, compulsions etc. But clinical features related to cognition, depression, and mood fluctuations, sleep disturbances may be more common in adult group. In view of PITANDS that occurs following infections with streptococci (81\%) \& other infections (19\%) like influenza, Mycoplasma, Varicella (chickenpox) \& Lyme disease ${ }^{1,4,5}$, we propose the existence of a post-streptococcal neuropsychiatric syndrome in adults which may be in other words a fractionated PANDAS or PANS in pediatric age group.

\section{References}

[1] Susan E. Swedo, James F. Leckman and Noel R. Rose, From Research Subgroup to Clinical Syndrome: Modifying the PANDAS Criteria to Describe PANS (Pediatric Acute-onset Neuropsychiatric Syndrome), Pediatr Therapeut 2012, vol 2 issue 2, page 1-8.

[2] Susan E. Swedo, M.D., Henrietta L. Leonard, M.D., Marjorie Garvey, M.D.,Barbara Mittleman, M.D., Albert J. Allen, M.D., Ph.D., Susan Perlmutter, M.D.,Lorraine Lougee, L.C.S.W., Sara Dow, B.A., Jason Zamkoff, B.A., and Billinda K. Dubbert, M.S.N Pediatric Autoimmune Neuropsychiatric Disorders Associated With Streptococcal Infections: Clinical Description of the First 50 Cases, Am J Psychiatry 155:2, February 1998, 264-71.

[3] Sara M Bodner, Syed A Morshed, Bradley S Peterson, The question of PANDAS in adults, Biological Psychiatry 1 May 2001 volume 49, Issue 9, page 807-810

[4] PANDAS Network.org. PANDAS/ANS Statistics 2012. Accessed at pandasnetwork.org/wp content/uploads/2012/08/Statistics.pdf.

[5] Hanna Rhee, Daniel J Cameron, Lyme disease and paediatric autoimmune neuropsychiatric disorders associated with streptococcal infections (PANDAS): an overview, International Journal of General Medicine, 21 February 2012, page 163-174.

[6] Church AJ, Dale RC, Antistreptolysin-O titers: implications for adult PANDAS. Pediatric autoimmune neuropsychiatric disorders associated with streptococcal infections, Am J Psychiatry. 2002 Feb; 159(2):320.

[7] Swedo SE, Sydenham's chorea. A model for childhood autoimmune neuropsychiatric disorders, JAMA, 1994 Dec 14; 272(22):17881791

[8] Kingston D, Glynn LE, Anti-streptococcal antibodies reacting with brain tissue. I. Immunofluourescent studies, Br $J$ Exp Pathol. 1976 Feb; 57(1):114-28

[9] Pavone P, Bianchini R, Parano E, Incorpora G, Rizzo R, Mazzone L, Trifiletti RR, Anti-brain antibodies in PANDAS versus uncomplicated streptococcal infection, Pediatr Neurol, 2004 Feb;30(2):107-10.

[10] Musser JM, Hauser AR, Kim MH, Schlievert PM, Nelson K, Selander RK. Streptococcus pyogenes causing toxic-shock-like syndrome and other invasive diseases: clonal diversity and pyrogenic exotoxin expression. Proc Natl Acad Sci U S A. Apr 1 1991; 88(7):2668-72.

[11] Cunningham MW. Pathogenesis of group A streptococcal infections. Clin Microbiol Rev. Jul 2000; 13(3):470-511.

[12] Snider LA, Swedo SE, PANDAS: current status and directions for research, Molecular Psychiatry, (2004) Oct; 9:900-907

[13] Singer HS, Loiselle CR, Lee O, Minzer K, Swedo S, Grus FH, Anti-basal ganglia antibodies in PANDAS, Mov Disord. 2004 Apr; 19(4):406-15.

[14] Asbahr FR, Negrão AB, Gentil V, Zanetta DM, da Paz JA, Marques-Dias MJ, Kiss MH, Obsessive-compulsive and related symptoms in children and adolescents with rheumatic fever with and without chorea: a prospective 6-month study, Am J Psychiatry. 1998; 155 (8): 1122.

[15] Snider LA, Lougee L, Slattery M, Grant P, Swedo SE, Biol. Psychiatry, Antibiotic prophylaxis with azithromycin or penicillin for childhood-onset neuropsychiatric disorders, 2005 Apr 1;57(7):788-792.

[16] Garvey MA, Perlmutter SJ, Allen AJ, Hamburger S, Lougee L, Leonard HL, Witowski ME, Dubbert B, Swedo SE, A pilot study of penicillin prophylaxis for neuropsychiatric exacerbations triggered by streptococcal infections, Biol Psychiatry. 1999 Jun 15; 45(12):1564-1571

[17] Lisa A. Snider, M.D. and Susan E. Swedo, M.D, Childhood-Onset Obsessive-Compulsive Disorder and Tic Disorders: Case Report and Literature Review, JOURNAL OF CHILD AND ADOLESCENT PSYCHOPHARMACOLOGY, Volume 13, Supplement 1, 2003, S81-S88.

[18] Susan J Perlmutter, Susan F Leitman, Marjorie A Garvey, Susan Hamburger, Elad Feldman, Henrietta L Leonard,Susan E Swedo, Therapeutic plasma exchange and intravenous immunoglobulin for obsessive-compulsive disorder and tic disorders in childhood, THE LANCET, Vol 354, October 2, 1999.

[19] Nabil Kitchener, Paediatric Autoimmune Neuropsychiatric Disorders Associated with Streptococcal infections (PANDAS), The International Journal of Child. Neuropsychiatry, 2005, 2(2): 195-202 